\title{
Analysis on the influence of grain size and grain layer thickness on the sorption kinetics of grained wood at low relative humidity with the use of water vapour sorption experiments
}

\author{
Alexander $\operatorname{Murr}^{1}$ (D) Roman Lackner ${ }^{1}$
}

Received: 6 June 2017 / Published online: 16 March 2018

(C) The Author(s) 2018

\begin{abstract}
Water vapour sorption (WVS) experiments on grained Norway spruce wood (Picea abies) at low relative humidities were carried out to test the influence of grain size and grain layer thickness on the sorption kinetics. Samples were compared under identical climatic conditions (i.e. humidity and temperature), and the kinetic behaviour was analysed with selected modelling approaches existing in the literature. Both, grain size and grain layer thickness influenced the initial kinetics, with the latter showing a larger impact. This confirms the notion of a transport limited initial mass increase with diffusion of water vapour $/ \mathrm{H}_{2} \mathrm{O}$-molecules to the sorption sites being a possible candidate. In contrast, the long-time behaviour was only slightly affected, supporting the concept of a relaxation and reorganisation dominated long-time behaviour. An analysis on the WVS kinetics of cut and grained wood with comparable sample material has further shown a very similar behaviour, which allows to draw some conclusions for cut wood. Regarding the modelling approaches, the parallel exponential kinetics model provided the best fitting results as the predictive models could not properly capture the split-up for a variation in grain size or grain layer thickness.
\end{abstract}

\section{Introduction}

Water vapour sorption experiments are a frequently used method to get information on the transport of water vapour through the macrostructure of wood and its subsequent sorption behaviour (e.g. Christensen and Kelsey 1959; Wadsö 1993; Eitelberger et al. 2011). Samples were exposed to variable climate conditions and

Alexander Murr

alexander.murr@gmx.at

1 Unit of Material Technology, University of Innsbruck, Technikerstraße 13, 6020 Innsbruck, Austria 
Table 1 Range of diffusion coefficients for water vapour $\left(D_{i}\right)$ and bound water $\left(D_{i, \mathrm{cw}}\right)$ in spruce wood in $\mathrm{m}^{2} / \mathrm{s}$ at room temperature

\begin{tabular}{llll}
\hline & Longitudinal $(L)$ & Radial $(R)$ & Tangential $(T)$ \\
\hline$D_{i}$ & $2.6 \cdot 10^{-5}$ & $1.9 \cdot 10^{-6}$ & $1.3 \cdot 10^{-6}$ \\
$D_{i, \mathrm{cw}}$ & $10^{-12} \cdots 10^{-10}$ & $0.5 \cdot D_{L, \mathrm{cw}}$ & $0.3 \cdot D_{L, \mathrm{cw}}$ \\
\hline
\end{tabular}

Values from Siau (1984) and Eitelberger et al. (2011)

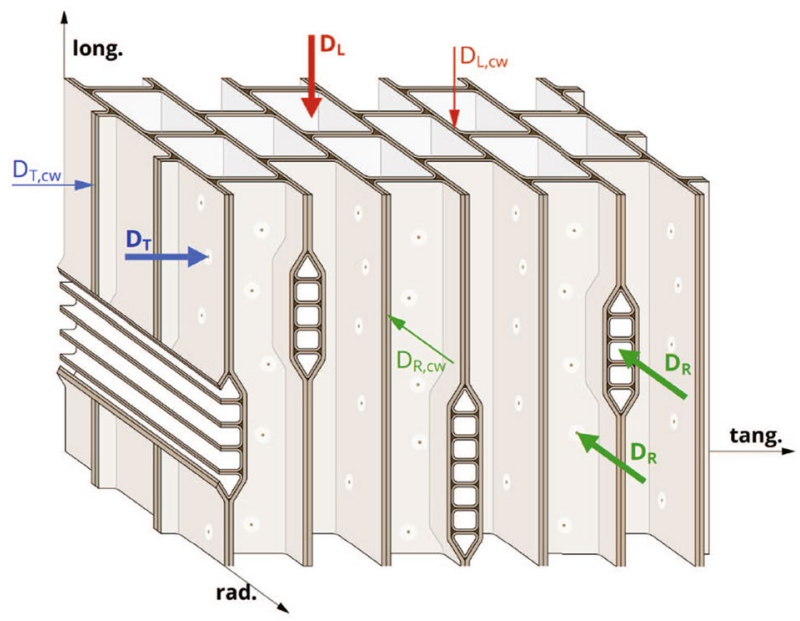

Fig. 1 Simplified structure of softwood with diffusion coefficients in the three material directions

weighted continuously. The amount of bound water is measured directly without any further assumptions. Experimental investigations on the sorption kinetics of wood have been performed for various wood species (e.g. Zaihan et al. 2009), sample sizes (e.g. Eitelberger and Svensson 2012) and also for grained wood (e.g. Hill et al. 2010b). However, an investigation on the influence of grain size and grain layer thickness on the WVS behaviour as well as an appropriate comparison between cut and grained wood seems to be missing.

A challenging part in the interpretation of such dynamic sorption experiments is the separation of transport processes and processes concerning the structural relaxation and reorganisation inside the cell wall (Popescu and Hill 2013). According to the long research activities during the last decades, many attempts were done to explain the WVS kinetics of wood (see e.g. Avramidis and Siau 1987; Krabbenhoft and Damkilde 2004; Hill et al. 2011). In contrast to descriptions of equilibrium values (e.g. sorption isotherms), care has to be taken about both, transport and relaxation processes. The former ones can be divided into a transport of water vapour to the sample surface, through the macrostructure and inside the cell wall (see Fig. 1). Average values for the diffusion coefficients are given in Table 1, with $D_{L}>D_{R}>D_{T}$ for the macrostructure (i.e. lumens, pits) and $D_{L, \mathrm{cw}}>D_{R, \mathrm{cw}}>D_{T, \mathrm{cw}}$ 
for the transport inside the cell wall (Siau 1984; Eitelberger et al. 2011). Apart from the particular binding mechanism, cell wall material acts as a sink/source where water molecules are getting bound, slowing down the effective transport (see Crank 1975). In contrast, relaxation and reorganisation processes include all kinds of phenomena regarding structural changes (macro to submicro-range), molecular rearrangements and the generation of additional sorption sites (see e.g. Engelund et al. 2012 and references therein). Conceptions of a two-stage process with a diffusioncontrolled first stage and a diffusion- and stress- or relaxation-controlled second stage were already discussed in the past (see e.g. Christensen 1967) as well as various other theories, like the two independent parallel processes described by the PEK model (e.g. Hill et al. 2010b). A more recent work has further shown three independent parallel processes (TEK-model) to be necessary for an accurate description of the sorption kinetics of wood (Glass et al. 2017). Thus, the difficult task is to figure out, which kind of information on the relaxation/binding processes could be extracted with the use of ordinary WVS experiments and to which extent the observed kinetics are influenced by any transport processes.

In the present study, the influence of grain size and grain layer thickness on the WVS behaviour of grained wood at low RH is investigated and analysed with the use of three different sorption kinetic models. These modelling approaches to describe and simulate the mass change of wood for a step change in $\mathrm{RH}$ are presented in "Existing models" section. The experimental set-ups are given in "Material and methods" section, including details on the performed simulations (e.g. geometry and used parameters). "Results and discussion" section represents the experimental results, showing the similarity between sliced and grained wood ("Comparison of sliced and grained wood" section), the effect of grain size ("Comparison of various grain sizes (monolayer)" section) as well as the influence of grain layer thickness ("Comparison of multilayer experiments" section). The objective of this study is thus to provide a detailed analysis on the WVS behaviour of grained wood and to get more information on the processes dominating the sorption kinetics at low relative humidity. Additionally, the differences between various modelling approaches and their application on grained wood will be discussed.

\section{Existing models}

Regarding the mentioned transport and relaxation processes, three types of cases can be distinguished to model the WVS behaviour of wood:

(1) Relaxation dominated case

(2) Transport dominated case

(3) Mixed case, where transport and relaxation processes are comparable

Accordingly, the various modelling approaches in the literature can be separated into three cases. A similar classification can be found in Hill et al. (2011) for the diffusion behaviour of swelling polymers. In the following, three modelling approaches 
are presented, covering all of the before-mentioned cases. They are based on different ideas and were used frequently in recent years. Further, the given models range from a heuristic relationship (PEK model) to a semi-predictive (bound water diffusion model) and a predictive approach (coupled diffusion model).

\section{Parallel exponential kinetics (PEK) model}

A rather empirical approach to describe the WVS behaviour of wood is given by the PEK model, where two exponential terms characterise the sorption process:

$$
\begin{aligned}
M C(t)= & M C_{1}\left(1-\exp \left(-t / \tau_{1}\right)\right) \\
& +M C_{2}\left(1-\exp \left(-t / \tau_{2}\right)\right) .
\end{aligned}
$$

The increase or decrease of sample moisture content $M C(t)$ for a step change of RH is represented by a fast and slow process with characteristic times $\tau_{1,2}$ and a corresponding moisture content $M C_{1,2}$. No information on the sample geometry (i.e. no spatial dependence) is required. This model has already been used for several materials (e.g. Kohler et al. 2003), and various interpretations concerning the two processes have been given (e.g. Okubayashi et al. 2004). The application to wood was done by Hill et al. (2010a) using small sample sizes. An interpretation in terms of a mechanical model has been given in Hill et al. (2011) assuming sorption kinetics being dominated by the viscoelastic behaviour of the cell wall (relaxation dominated case). Consequently, the fast and slow process are described each with a Kelvin-Voigt element

$$
\varepsilon(t)=\frac{\sigma_{0}}{E}(1-\exp (-t / \tau))
$$

where $\varepsilon(t)$ denotes the strain, $\sigma_{0}$ the stress and $E$ the elastic modulus. The time constant $\tau$ is given as the ratio of viscosity and elastic modulus, $\tau=\eta / E$. A linear relation between changes in mass (during adsorption/desorption) and cell wall volume is assumed. For the connection between stress and changes in $\mathrm{RH}$, a relationship for the swelling pressure exerted by an elastic gel is used.

Later, a study on naturally aged lime wood has shown significant differences in the behaviour between the fast and slow process (Popescu and Hill 2013). An investigation on the pseudo-isotherms given by a separation of the two processes led then to a reinterpretation (mixed case): The slow process is suggested to be a relaxation limited process, whereas the fast process is associated with a diffusion limited phenomenon (Popescu et al. 2014). A further attribution of the fast process in terms of the linear driving force model has been given by Popescu et al. (2015), which has already been used for activated carbon (e.g. Foley et al. 1997). This phenomenological model describes diffusion and adsorption of particles with a pseudo-first-order mass transfer relationship (see e.g. Alpay and Scott 1992),

$$
\frac{\mathrm{d} M(t)}{\mathrm{d} t}=k\left(M_{\infty}-M(t)\right)
$$


with the mass $M(t)$ and its equilibrium value $M_{\infty}$. Depending on the interpretation of Eq. (3), the proportionality factor $k$ can be defined either as a mass transfer coefficient or as a first-order reaction rate. Transport and relaxation processes seem thus necessary for modelling the WVS behaviour of wood. However, a clear and unique interpretation of the two processes described with the PEK model seems to be still missing (Himmel and Mai 2016). Further investigations might thus be useful to identify the nature of these processes.

\section{Bound water diffusion model}

An attempt to model the mass change of wood in WVS experiments as a single diffusive process has been given by Olek et al. (2005). The local bound water content $m=m(x, t)$ is assumed to follow a diffusion equation,

$$
\frac{\partial m}{\partial t}=\frac{\partial}{\partial x}\left(D_{b} \frac{\partial m}{\partial x}\right)
$$

and the diffusion coefficient is modelled to be either constant, $D_{b}=D_{0}$ or variable (Olek and Weres 2007) with the global bound water content $M=M(t)$,

$$
\begin{gathered}
D_{b}=D_{0} \exp (-a M(t)) \\
D_{b}=D_{0} \exp \left(-a M(t)-b M(t)^{2}\right) .
\end{gathered}
$$

$D_{0}, a$ and $b$ are estimated coefficients and for the latter two no physical explanation has been given. For the boundary condition, a flux is defined as:

$$
-D_{b} \frac{\partial m}{\partial x}=\sigma\left(m-M_{\infty}\right)
$$

with $\sigma$ being a surface emission coefficient and $M_{\infty}$ the equilibrium bound water content (transport dominated case). In order to match the local bound water content $\left(\operatorname{dim} m(t) \equiv \mathrm{kg} / \mathrm{kg}_{\text {dry }}\right)$ with the global bound water content (dim $M(t) \equiv \mathrm{kg} /$ $\left.\mathrm{kg}_{\mathrm{dry}}\right)$, the latter one has to be defined as $M(t):=1 / L \int_{0}^{L} m(x, t) \mathrm{d} x$, with $\mathrm{L}$ being the sample thickness. As a consequence of Eq. (7) the proximity to equilibrium at the surface restricts the flux inside the sample. As many experiments have shown the sorption kinetics of wood cannot be described by a simple diffusion equation (e.g. Christensen and Kelsey 1959; Wadsö 1993), a variable diffusion coefficient was used in this approach to model this so-called non-Fickian behaviour.

A modification on the equilibrium bound water content $M_{\infty}$ was later introduced by Olek et al. (2011) to account for additional sorption sites due to any relaxation and reorganisation effects during the sorption process,

$$
M_{\infty}(t)=c+d(1-\exp (-t / \tau)) .
$$

Here, the coefficient $c$ specifies the maximum moisture uptake before relaxation and $d$ represents the additional amount available after relaxation and reorganisation 
processes took place. There are thus fast accessible sorption sites and sites which need a certain relaxation time $\tau$ to be available (mixed case). This idea on a creation of new sorption sites seems reasonable for swelling materials and has already been mentioned by many authors (see e.g. Hartley et al. 1992). Regarding the non-Fickian behaviour, it was emphasised by Olek et al. (2011) the modified boundary condition with Eq. (8) yields more adequate results than the moisture-dependent diffusion coefficient.

\section{Coupled diffusion model}

A prominent model which might be assigned to any category (depending on its interpretation and the used sample thickness) is the coupled diffusion model of Krabbenhoft and Damkilde (2004). A set of two diffusion equations was proposed which are coupled by a heuristic sorption term. The transport of water vapour through the lumen is considered to be independent from the transport of bound water in the cell walls (with concentration $c_{b}$ ). A conversion of water vapour concentration $c_{v}$ to partial gas pressure $p_{v}=p_{v}\left(c_{v}\right)$ was done with the ideal gas law. Both processes are assumed to be diffusive,

$$
\begin{gathered}
\varphi \frac{\partial p_{v}}{\partial t}=\frac{\partial}{\partial x}\left(\varphi D_{v} \frac{\partial p_{v}}{\partial x}\right)-\dot{s} \frac{R T}{M_{w}} \\
\frac{\partial c_{b}}{\partial t}=\frac{\partial}{\partial x}\left(D_{b} \frac{\partial c_{b}}{\partial x}\right)+\dot{s}
\end{gathered}
$$

and an exchange is given by the coupling term $\dot{s}$. Here, $\varphi$ denotes porosity, $R$ is the gas constant, $T$ the temperature and $M_{w}$ the molecular weight of water. It has to be mentioned in their original work that the coefficients $\frac{R T}{M_{w}}$ and $\varphi$ on the right-hand side

of Eq. (9) have been omitted as already discussed in Frandsen et al. (2007). The sorption term has been formulated similar to a surface evaporation process,

$$
\dot{s}=h\left(p_{v}-p_{b}\right)
$$

with an equivalent equilibrium vapour pressure $p_{b}=p_{b}\left(c_{b}\right)$. This value has to be back calculated along the sorption isotherm and corresponds to the given (local) bound water concentration $c_{b}$. The proportionality factor $h$ was constructed to capture the experimental results,

$$
h=a_{1} \exp \left(-a_{2}\left(\frac{p_{b}}{p_{v}}\right)^{a_{3}}\right)+a_{4}
$$

and describes a variable surface evaporation. Thus, the sorption term is used to describe the non-Fickian behaviour in this approach. 
A modification of Eq. (12) has been given by Frandsen et al. (2007) with the coefficients $a_{i}$ depending continuously on relative humidity:

$$
a_{i}=a_{i 1} \exp \left(a_{i 2} R H\right)+a_{i 3} \exp \left(a_{i 4} R H\right) .
$$

The coefficients were adjusted to the experiments of Christensen (1965), where almost identical sorption kinetics were found for $20 \mu \mathrm{m}$ and $1 \mathrm{~mm}$ thick samples. In both cases, a physical interpretation of the proportionality factor and its coefficients seems to be difficult and is still missing.

Eitelberger et al. (2011) formulated a model without using a fitted function for the sorption term,

$$
\dot{s}=\alpha\left(\rho_{\mathrm{cw}} M C-c_{b}\right)
$$

with the dry density of the cell wall $\left(\rho_{\mathrm{cw}}\right)$ and the equilibrium moisture content $(M C)$. The proportionality factor $\alpha$ depends on the cell wall diffusion coefficient and on a geometry factor of a single tracheid cell. Additionally, a third differential equation for the energy (or temperature) was added but without including any mechanical energy for the swelling/shrinkage of wood. Any kind of relaxation or reorganisation processes have not been included in this model.

\section{Material and methods}

\section{Sample preparation}

Norway spruce wood (Picea abies) grown $1200 \mathrm{~m}$ above sea level in West Austria was used. A board from the lower third of the stem was cut along the longitudinal direction. It went through the centre of the tree in order to determine the annual position. Prior to the manipulation, the board was stored at $T \approx 22{ }^{\circ} \mathrm{C}$ and a relative humidity of about $40 \%$ for 3 months. Wood samples were taken without using the first eight and last ten annual rings. Sliced samples were used from both, circular saw cut and microtomed. For the circular saw cut samples, slices with a cross section of $43 \mathrm{~mm} \times 43 \mathrm{~mm}( \pm 0.5 \mathrm{~mm})$ and a thickness of $0.5 \mathrm{~mm}( \pm 0.1 \mathrm{~mm})$ were used. One representative slice in longitudinal direction (i.e. the surface is perpendicular to the tracheid cells) was chosen. Attention has to be given in order not to generate too much heat during the cutting process, as the cell wall structure in the vicinity might be affected. Hence, a sharp saw blade with alternative top bevel teeth and low rake angle was used. The feed rate and the number of revolutions were adjusted in order to minimise the heat production. It might also be considered to increase the moisture content of wood, as the specific heat capacity and heat conductivity are increasing while the strength is decreasing with moisture content. In the case of the microtomed samples, slices with a cross section of $10 \mathrm{~mm} \times 10 \mathrm{~mm}( \pm 0.5 \mathrm{~mm})$ and a thickness of $25 \mu \mathrm{m}( \pm 1 \mu \mathrm{m})$ were used. About 20 slices were taken in the longitudinal direction. For the grained samples, a large piece of wood from the same board was ground separately with three double-cut files and a P100 sanding paper (average particle size diameter $\approx 162 \mu \mathrm{m}$ ). An average over many annual rings and tracheid cells could thus be provided. It might be mentioned to instead use a mill to 
obtain a better repeatability of the ground sample material and probably to provide a better integrity of the cell wall structure. Afterwards, the grained wood was double sieved with a set of 7 analysis sieves to obtain grain size distributions between $1 \mathrm{~mm}$ and $20 \mu \mathrm{m}$. Hence, length was varied up to a factor of 20 between the largest and smallest grains. For the smallest grain size distribution $(20-63 \mu \mathrm{m})$, it is possible that it consists partly of pure cell wall material, as the mean diameter of earlywood tracheid cells was measured to be between $30-40 \mu \mathrm{m}$. A laser diffraction analysis on the sieved samples has been done to cross-check the used grain size fractions. In the following experimental set-ups, a circular saw cut slice, microtomed slices and three grain size fractions with various sample masses were used (see Table 2).

\section{Measuring apparatus}

To measure mass change (i.e. sorption kinetics), wood samples were exposed to various humidities and weighted continuously. Therefore, the water vapour sorption system SPSx-1 $\mu$ (ProUmid GmbH, Germany) was used. This apparatus is composed of a measuring chamber $(34 \mathrm{~cm} \times 43 \mathrm{~cm} \times 7 \mathrm{~cm})$, a rotating plate to hold 11 aluminium sample bowls with $51 \mathrm{~mm}$ diameter and a micro-balance with a reproducibility of $\pm 10 \mu \mathrm{g}$ (Fig. 2). Sample bowls are placed automatically on the balance to record the mass change of each probe staggered. Additionally, a reference bowl is weighted each time before the first and after the last sample to correct the water uptake of the sample bowls and a possible balance drift. This was done with the assumption of a linear drift between the first and the last sample. As a working fluid dry air is used and the humidity is controlled by the moistening unit with purified water. Consequently, either dry $(\mathrm{RH}=0.1 \%)$ or wet air $(\mathrm{RH} \approx 99 \%)$ is blown into the measuring chamber and two fans distribute the resulting air-mixture with a velocity of $0.3 \mathrm{~m} / \mathrm{s}$. Higher velocities could already remove the microtomed and grained particles out of

Table 2 Classification of sliced wood $(A 1, A 4)$ and grained wood by minimal-maximal particle size and sample mass (at $\mathrm{RH}=20 \%$ ) for the experimental investigations

\begin{tabular}{lll}
\hline Sample mass $(\mathrm{mg})$ & Particle size & Label \\
\hline 365 & $0.5 \mathrm{~mm} \times 43 \mathrm{~mm} \times 43 \mathrm{~mm}$ & $A 1$ \\
200 & $125-250 \mu \mathrm{m}$ & $A 2$ \\
400 & $125-250 \mu \mathrm{m}$ & $A 3$ \\
22 & $25 \mu \mathrm{m} \times 10 \mathrm{~mm} \times 10 \mathrm{~mm}$ & $A 4$ \\
22 & $20-63 \mu \mathrm{m}$ & $A 5$ \\
20 & $20-63 \mu \mathrm{m}$ & $B 1$ \\
20 & $500-1000 \mu \mathrm{m}$ & $B 2$ \\
200 & $20-63 \mu \mathrm{m}$ & $C 1$ \\
200 & $125-250 \mu \mathrm{m}$ & $C 2$ \\
200 & $500-1000 \mu \mathrm{m}$ & $C 3$ \\
200 & $20-63 \mu \mathrm{m}$ & $C 4$ \\
400 & $20-63 \mu \mathrm{m}$ & $C 5$ \\
600 & $20-63 \mu \mathrm{m}$ & $C 6$ \\
800 & $20-63 \mu \mathrm{m}$ & $C 7$ \\
\hline
\end{tabular}




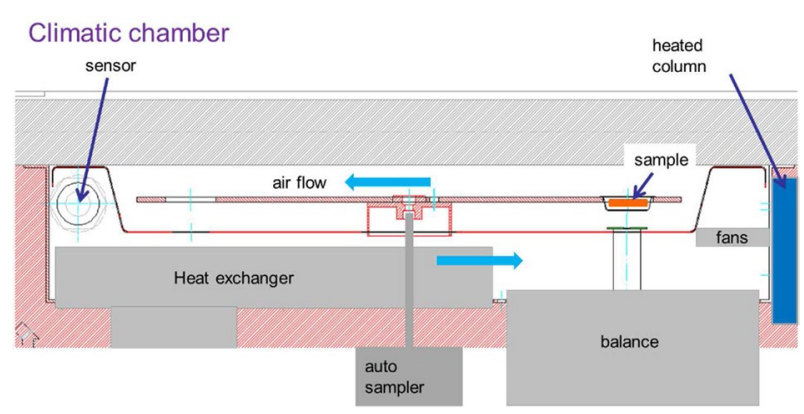

Fig. 2 Detail drawing of the water vapour sorption measuring system. Source: ProUmid

the bowls. Fans and humidification were turned off when a sample is placed on the balance.

A change of relative humidity at $T=25^{\circ} \mathrm{C}$ took about $1 \mathrm{~min}$ for the experiments with $\Delta \mathrm{RH}=5 \%$ and $\Delta \mathrm{RH}=10 \%$ with a deviation below $1 \%$. A deviation less than $\pm 0.3 \%$ to the pre-set humidity values could be ensured except for $\mathrm{RH} \leq 1 \%$ $( \pm 0.1 \%)$. The achievable humidity ranges from $\mathrm{RH}=0.1 \%$ to $\mathrm{RH}=95 \%$ for $T=25^{\circ} \mathrm{C}$. Variations of temperature were below $\pm 0.1^{\circ} \mathrm{C}$. To achieve a stabilised climate for the samples, measurement cycles were executed in an 8 min interval.

\section{Experimental set-up}

Cleaned sample bowls were placed on the rotating plate and tared at $\mathrm{RH}=20 \%$ and $T=25^{\circ} \mathrm{C}$. Sliced and grained samples with a weight according to Table 2 were poured in the sample bowls. The surface was carefully flattened with a spatula in order to avoid compression among the various grain sizes. Minimum sample mass was chosen at $20 \mathrm{mg}$ to obtain a reasonable mass resolution. Sample masses were weighted out with an accuracy of $\pm 0.2 \mathrm{mg}$ for approximately $20 \mathrm{mg}$ and $\pm 1 \mathrm{mg}$ for a mass $\geq 200 \mathrm{mg}$. The used step size in RH was chosen to be $\Delta \mathrm{RH}=5 \%$ in order to reduce the influence of the non-instantaneous step change on the sorption kinetics. Temperature was kept constant at $25^{\circ} \mathrm{C}$ during the whole step. Only for experiments on the lower resolution scale, step size was chosen at $\Delta \mathrm{RH}=10 \%$ in order to increase the signal-to-noise $(S / N)$ ratio by a larger amount of absolut water uptake. In the following, experimental set-ups for three different investigations on the sorption behaviour of grained wood is given.

\section{Comparison of sliced and grained wood}

To analyse the differences in the kinetic behaviour of sliced and grained wood, an experimental set-up with a sample thickness in the $\mathrm{mm}$ and $\mu \mathrm{m}$-range was used. Care was taken regarding sample thickness and weight (i.e. amount of sorption sites per unit cross section) as water vapour has to be transported to and through the sample material. For the comparison in the mm-range, a slice of cut wood in 
longitudinal direction with $0.5 \mathrm{~mm}$ thickness and a mass of $365 \mathrm{mg}(A 1)$ was used. The sliced sample stood out of the sample bowl and was exposed to the surrounding RH on both sides. In contrast, grained samples were only exposed on the upper side (i.e. on one side) to RH, but had a higher accessibility based on the spherical shape. As the number of sorption sites is an important parameter for a diffusion process with a sink, grained wood samples with a mass of $200 \mathrm{mg}$ (A2) and $400 \mathrm{mg}$ (A3) were chosen. Grain size of 125-250 $\mu \mathrm{m}$ was used as pre-testings with other grain sizes have shown a similar behaviour (cf. "Comparison of multilayer experiments" section). The corresponding grain layer thickness can be estimated to 0.7 and $1.4 \mathrm{~mm}$ (Eq. 16). Cross-sectional areas between the cut and grained samples were comparable. For the comparison in the $\mu \mathrm{m}$-range, sample mass was chosen to be small enough to avoid overlapping of the slices or grains but still as high to provide an acceptable $S / N$-ratio with the given sorption analyser. Hence, a sample mass of $22 \mathrm{mg}$ was used. About 20 slices of the microtomed samples with $25 \mu \mathrm{m}$ thickness $(A 4)$ were needed and only a few were partly overlapping. In contrast to the comparison of sliced and grained wood in the mm-range, the microtomed and grained samples were both exposed to RH in a similar manner. Therefore, a similar thickness appeared reasonable and grain size was chosen slightly larger as it offers a faster accessibility due to the spherical geometry. The grained sample was consequently chosen with a grain size of $20-63 \mu \mathrm{m}$ and equal mass (A5). Further, a similar cross-sectional area of approximately $19 \mathrm{~cm}^{2}$ could be reached.

\section{Comparison of grain sizes (monolayer)}

To figure out possible differences in the WVS behaviour of various grain sizes, grained wood with a single grain layer (monolayer) and equal sample mass was used. Tests on monolayer experiments with five different grain size distributions were performed in advance to estimate the differences in their sorption kinetics. To point out the differences more clearly, an experiment with only two samples was performed. Thus, grained wood with a grain size of 20-63 $\mu \mathrm{m}(B 1)$ and 500-1000 $\mu \mathrm{m}(B 2)$ and a mass of $20 \mathrm{mg}$ was used. With this set-up, a reduction of the measurement cycle time from 8 to $5 \mathrm{~min}$ could be achieved, corresponding to a faster time resolution of the measuring device. Similar to the comparison of sliced and grained wood in the $\mu \mathrm{m}$-range, sample mass in this set-up is on the lower range limit of the sorption measuring system. Hence, the $S / N$-ratio is rather low and thus step size in RH was chosen to a larger value.

\section{Comparison of multilayer experiments}

In order to determine the effect of layering of grains on the sorption kinetics of grained wood, two experimental set-ups with multiple grain layers (multilayer) were used. To analyse the influence of grain sizes, multilayer experiments with three different grain sizes and equal mass were compared. Grain size distributions were chosen to 20-63 $\mu \mathrm{m}(C 1), 125-250 \mu \mathrm{m}(C 2)$ and 500-1000 $\mu \mathrm{m}(C 3)$ with a sample mass of $200 \mathrm{mg}$. As all samples do have the same amount of sorption sites, any additional effects caused by an external water vapour supply limitation were minimised. Other 
sample masses were tested in advance showing a similar behaviour. With these multilayer experiments possible differences could be distinguished more accurately than for the monolayer experiments, as the relative error decreases with increasing sample mass. According to Eq. 16, the given sample mass is equivalent to a grain layer thickness of $0.67 \mathrm{~mm}(C 1), 0.71 \mathrm{~mm}(C 2)$ and $0.84 \mathrm{~mm}(C 3)$. For the determination of the influence of grain layer thickness (i.e. amount of sorption sites per unit cross section), grained wood with multiple grain layers and a single grain size of 20-63 $\mu \mathrm{m}$ was used. Sample mass was chosen to $200 \mathrm{mg}(C 4), 400 \mathrm{mg}$ (C5), $600 \mathrm{mg}(C 6)$ and $800 \mathrm{mg}(C 7)$, respectively. These sample masses correspond to a grain layer thickness (cf. Eq. 16) of $0.7 \mathrm{~mm}(C 4), 1.3 \mathrm{~mm}(C 5), 2.0 \mathrm{~mm}(C 6)$ and $2.7 \mathrm{~mm}(C 7)$.

Prior to testing, samples were conditioned for about $24 \mathrm{~h}$ at $\mathrm{RH}=0.1 \%$ and $T=25^{\circ} \mathrm{C}$ in the measuring chamber. A complete run-stepwise from 0.1 to $95 \%$ $\mathrm{RH}$, followed by the reverse order decreasing sequence-was performed in advance to provide a better reproducibility. This fact was observed in earlier experiments and was also reported in literature (see e.g. Popescu and Hill 2013). For each step, humidity was maintained within the given limits until the equilibrium condition (EC) was reached for all samples. The EC was defined as

$$
\frac{\mathrm{d}}{\mathrm{d} t}\left(\frac{m(t)}{m_{\min }}\right) \leq 0.02 \%
$$

in a 120 min period and was obtained by a reasonable compromise between equilibrium accuracy and measuring time. Here, $m(t)$ denotes the net sample mass and $m_{\min }$ its minimum value after preconditioning at $0.1 \% \mathrm{RH}$.

\section{Error estimation}

To determine the measurement error expected in the WVS experiments, three different sources have to be taken into consideration. For experiments with a sample mass in the order of $20 \mathrm{mg}$, the reproducibility of the balance $( \pm 10 \mu \mathrm{g})$ becomes important. With a total mass increase of approximately $520 \mu \mathrm{g}$ for a step change of $0 \rightarrow 10 \% \mathrm{RH}$, this error contributes with $2 \%$ to the mass increase of these samples. As the results are represented in relative changes of sample mass (cf. Eq. 17), the errors arising from balance reproducibility decrease with increasing sample mass. The second source of error which is more dominant at the beginning of the sorption process (i.e. the first measuring values) is caused by local and global fluctuations in humidity. This error has been estimated (on the basis of previous experiments) to be in the order of $\pm 20 \mu \mathrm{g}$ for fluctuations in the RH-step change between 0 and $10 \% \mathrm{RH}$ and contributes thus with $4 \%$ to the absolute mass increase. Again, samples with a larger thickness and a higher weight are less affected as both, diffusion and the amount of water molecules in the vapour fluctuation restrict the resulting impact. The third source of error arises from random disturbances of the balance caused by temporary vibrations that are transferred over the floor/building. Even though this error could be rather large, it affects usually only a single measuring value and can thus be easily detected. For the following results error bars 
were chosen according to the maximum value of the three mentioned errors which is appropriate to the particular experiment. It has to be noted, in the case of grained wood there is a large amount of single grains in each sample bowl, and thus, an average value for the mass increase is measured.

\section{Simulations}

The simulations of the mentioned models in "Existing models" section were done with the software Wolfram Mathematica 10.4. For the PEK model, the parameters were determined by a nonlinear model fit. As no spatial variables are included in this approach, sample geometry has not to be taken into account. In the case of the bound water diffusion model, Eqs. (4), (5), (7) and (8) were used without performing an inverse analysis as given in Olek et al. (2011). The bound water diffusion coefficient $\left(D_{b}\right)$, the surface emission coefficient $(\sigma)$ and the time constant $(\tau)$ were fitted to the experiment, while the remaining parameters were used as given for the lowest RH range in their work (Olek et al. 2011). For the coupled diffusion model, Eqs. (9), (10), (11), (12) and (13) were used, including all necessary parameters given in Frandsen et al. (2007) which were based on earlier experiments of sliced Klinki pine wood (Araucaria hunsteinii). No adjustment on the parameters was performed as this model serves to predict the WVS kinetics of wood.

Except for the PEK model, simulations were solved in 1D according to a sheet diffusion problem. The similarity between the measured sorption kinetics of cut and grained wood (see "Comparison of sliced and grained wood" section) makes this assumption somewhat feasible, even though the 1D treatment of transport processes through packed spheres seems to be too simplistic. Further, the grained samples with multiple grain layers do not have a continuous cell wall between the upper and lower boundary of the sample as in the case of cut wood. An application and interpretation of the bound water diffusion model seems thus to be questionable. For grained wood with a single grain layer (monolayer), a simulation of the given models for homogeneous spheres was done additionally, as effects of layering (e.g. parallel transport paths for water vapour) do not have to be considered in this case. These simulations can be seen as a direct test of the existing WVS modelling approaches for small sample sizes with equal weight but different size. The spherical solution will be used as an upper limit for the mass increase, since the diffusion process in the sphere is calculated uniformly with the water vapour diffusion coefficient for the longitudinal direction. On the contrary, the sheet solution serves the lower case limit (i.e. a too slow mass increase), where the grains are treated as a sheet with a thickness of twice the radius and without any transport in the transversal directions (Fig. 3). This could be done as all tracheid cells are opened and the diffusion in longitudinal direction is much faster than in radial or tangential direction (see Table 1).

\section{Estimation of grain layer thickness}

Samples were weighed out to a predefined weight in order to ensure the same amount of total sorption sites. As the grain layer thickness of the multilayer samples 


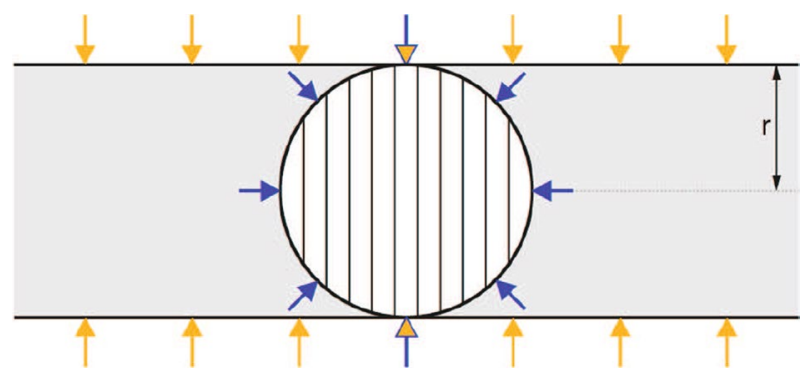

Fig. 3 Comparison of spherical and sheet diffusion indicating the upper (sphere) und lower limit (sheet) for the WVS kinetics of grained wood. Black lines inside the sphere indicate the lumen pathways for wood and $r$ denotes the radius of the sphere

could be measured only roughly, an estimation of their height based on the weight was preferred. Differences between the various grain sizes could thus be sufficiently resolved in order to perform the corresponding simulations. Congruent spherical particles with a hexagonal closed packing were assumed. Boundary effects were neglected as the radius of the spheres is much smaller than the radius of the sample bowl. Accordingly, the grain layer thickness for a certain grain size (with a given weight) can be calculated as

$$
H_{\mathrm{S}}=2 R(1-\sqrt{2 / 3})+12 \sqrt{2} \frac{M_{\mathrm{S}}}{d^{2} \pi^{2} \rho_{\mathrm{W}}}
$$

Here, $R$ denotes the radius of the spheres, $M_{\mathrm{S}}$ the weight of the multilayer sample, $d$ the diameter of the sample bowl and $\rho_{\mathrm{W}}$ the density of wood. With the use of a $30 \%$ larger radius for the grains ( $\rho_{\mathrm{W}}$ decreases accordingly), reasonable values could be obtained for the given mono- and multilayer sample thickness of the experiments.

\section{Results and discussion}

In the following, a number of water vapour sorption experiments are shown. Step changes in relative humidity of $0 \rightarrow 5 \% \mathrm{RH}$ or $0 \rightarrow 10 \% \mathrm{RH}$ were compared for adsorption. Pretests have shown very similar sorption kinetics for the two mentioned step sizes and justify the comparability of the given results (Fig. 4). The lower range of relative humidity was chosen, as the sorption kinetic curves seem to be dominated by transport processes in this range. Consequently, differences in the initial kinetic behaviour based on differences in sample geometry (thickness, mass) should be most dominant at low RH. At higher RH the relevance of relaxation and reorganisation processes become more dominant and a notable deviation from the diffusive behaviour could be noticed. Hence, it seems as if certain differences in the sorption kinetics among the samples will be reduced or even covered by the relaxation behaviour of wood in the mid and high $\mathrm{RH}$ range. Similar facts have also been mentioned in literature (see e.g. Christensen and Kelsey 1959). The increase in sample mass is 


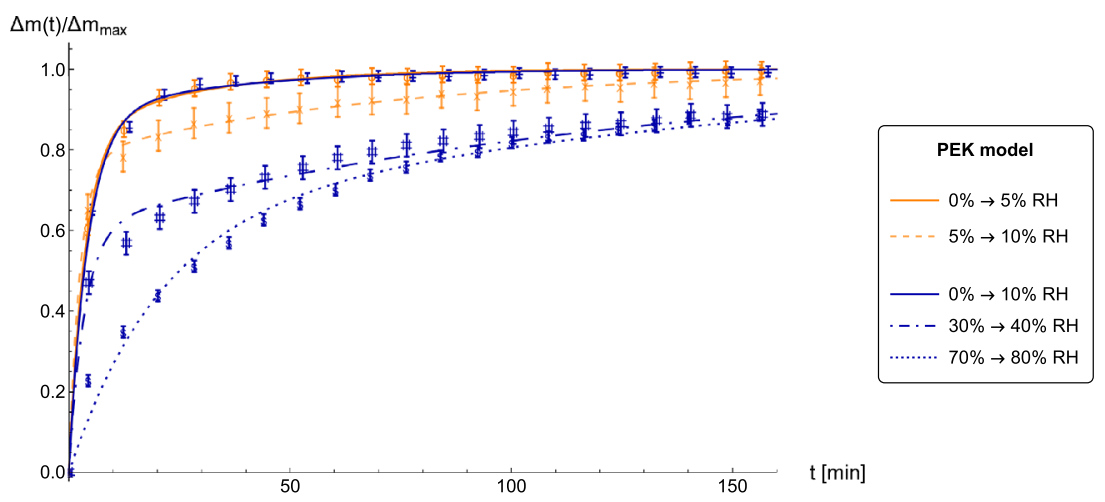

Fig. 4 Comparison of the WVS curves for grained wood $(20-63 \mu \mathrm{m})$ with a sample mass of $200 \mathrm{mg}$ for various step changes in RH. Experimental results (markers) were fitted with the PEK model (solid/ dashed/dot-dashed/dotted lines)

normalised to the maximum mass uptake in the given RH step. Samples with different masses can thus be compared by their relative changes in bound water content:

$$
\frac{\Delta m(t)}{\Delta m_{\max }}=\frac{m(t)-m(0)}{m\left(t_{\max }\right)-m(0)} .
$$

Each step change starts at $t=0$ and ends when the equilibrium conditions for all samples are fulfilled $\left(t_{\max }\right)$. For a better comparability, time axis was chosen with a scale according to the relevant domain although equilibrium (Eq. 15) was usually achieved at later times. As the comparison of sliced and grained wood is mainly to indicate the similarity of their WVS behaviour, measured data were only fitted with the PEK model.

\section{Comparison of sliced and grained wood}

\section{Comparison in the mm-range}

A comparison of the WVS kinetics of sliced and grained wood in the mm-range is shown in Fig. 5. Step change in $\mathrm{RH}$ was chosen to $0 \rightarrow 5 \%$ and temperature to $T=25^{\circ} \mathrm{C}$. There is an obvious split-up between the three samples in the beginning of the mass increase. The cut sample lies between the two grained ones, with the lower grain layer thickness $(200 \mathrm{mg})$ showing a faster kinetic than the higher one (400 mg). With an estimated sample thickness of $0.7 \mathrm{~mm}$ and $1.4 \mathrm{~mm}$, the grained samples are slightly smaller and larger than twice the thickness of the sliced wood. Weight of the cut sample was chosen to lie within the two grained samples, as a transport process with a larger sink takes more time for the mass increase than with a smaller sink (i.e. lesser amount of sorption sites). Concerning the long-time behaviour, all three samples do show a similar behaviour until equilibrium condition is 


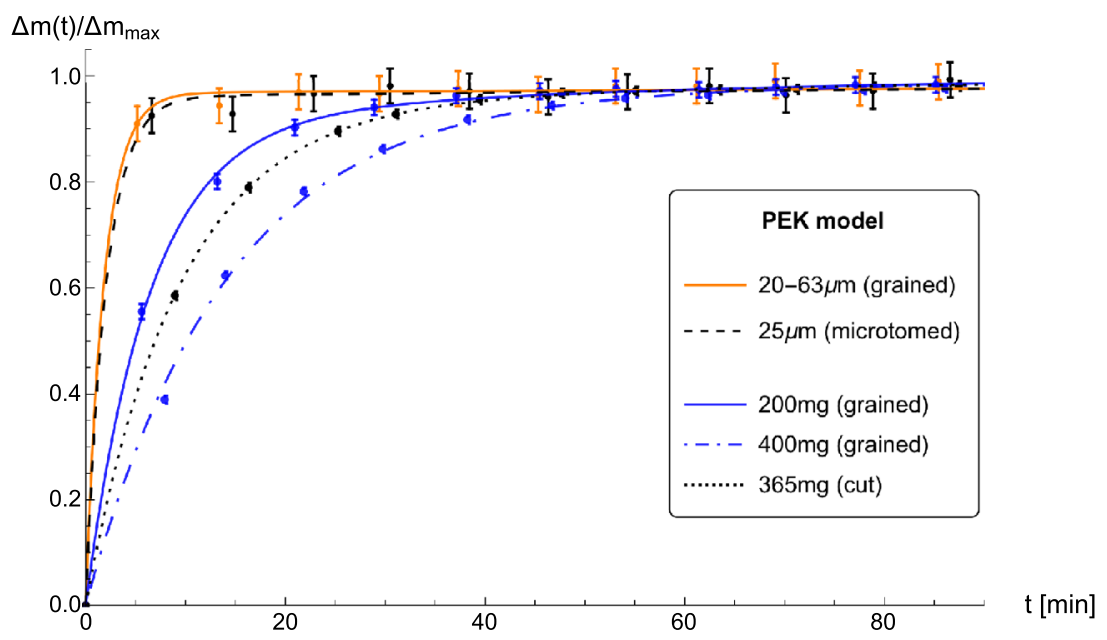

Fig. 5 Comparison of the WVS curves for sliced and grained wood in the mm-range and in the $\mu \mathrm{m}$-range. Experimental results (markers) were fitted with the PEK model (solid/dashed/dotted/dotdashed lines)

achieved (Eq. 15). Small differences can be seen with a steeper slope for an increasing sample weight. Expectedly, the long-time behaviour of the cut sample is more similar to the $200 \mathrm{mg}$ grained sample, as it was exposed to RH on both sides leading to a faster mass increase at the beginning (Fig. 5). The small mass increase for long measuring times seems to be influenced by the short-time behaviour, i.e. sample thickness, mass (per unit cross section) and the degree of exposure to RH. As a prior test with other materials has shown a different mass increase for the long-time behaviour, the sorption measuring system seems rather improbable to be responsible for this increase. Thus, it could be concluded, there is no essential difference in the WVS behaviour of cut and grained wood in the mm-range if a comparable sample material is used.

Regarding the PEK model, all three samples could be fitted within the error bars. Both, the time constants for the fast $\left(\tau_{1}\right)$ and slow process $\left(\tau_{2}\right)$ show an increasing tendency with sample weight as given in Table 3 . The difference at the beginning of mass increases between the two grained samples (with equal grain size but different layer thicknesses) in Fig. 5 is in contrast to the notion of both exponential processes in the PEK model being assigned to a mechanical origin (Eq. 2). More plausible would be the interpretation of a transport process at the beginning (fast process) and a relaxation process for long measuring times (slow process) similar to that suggested by Popescu et al. (2014).

\section{Comparison in the $\mu m$-range}

The results for the comparison of the WVS kinetics of sliced and grained wood in the $\mu \mathrm{m}$-range are given in Fig. 5, for a step change in RH of $0 \rightarrow 10 \%$ at $T=25^{\circ} \mathrm{C}$. Both samples show a similar behaviour, even though the sorption measuring system was too 
Table 3 Results for the fit parameters of the five experiments for the PEK model $\left(\tau_{1}, \tau_{2}\right)$ and the bound water diffusion model $\left(\tau, \sigma, D_{b}\right)$

\begin{tabular}{lrllll}
\hline Sample mass & $\tau_{1}(s)$ & $\tau_{2}\left(10^{3} \mathrm{~s}\right)$ & $\tau\left(10^{3} \mathrm{~s}\right)$ & $\sigma\left(10^{-6} \mathrm{~m} / \mathrm{s}\right)$ & $D_{b}\left(10^{-9} \mathrm{~m}^{2} / \mathrm{s}\right)$ \\
\hline $365 \mathrm{mg}(0.5 \mathrm{~mm})$ & 560 & 3.8 & & & \\
$200 \mathrm{mg}(125-250 \mu \mathrm{m})$ & 380 & 2.8 & & \\
$400 \mathrm{mg}(125-250 \mu \mathrm{m})$ & 830 & $5.3^{*}$ & & \\
$22 \mathrm{mg}(25 \mu \mathrm{m})$ & 120 & $11.3^{*}$ & & \\
$22 \mathrm{mg}(20-63 \mu \mathrm{m})$ & 110 & $18^{*}$ & & & \\
$20 \mathrm{mg}(20-63 \mu \mathrm{m})$ & 80 & $3.1^{*}$ & 1.4 & 1.2 & 1.6 \\
$20 \mathrm{mg}(500-1000 \mu \mathrm{m})$ & 130 & $1.7^{*}$ & 1.4 & 1.2 & 1.6 \\
$200 \mathrm{mg}(20-63 \mu \mathrm{m})$ & 280 & 3.8 & 3.3 & 3.0 & 1.6 \\
$200 \mathrm{mg}(125-250 \mu \mathrm{m})$ & 320 & 4.2 & 3.3 & 3.0 & 1.6 \\
$200 \mathrm{mg}(500-1000 \mu \mathrm{m})$ & 380 & 4.6 & 3.3 & 3.0 & 1.6 \\
$200 \mathrm{mg}(20-63 \mu \mathrm{m})$ & 220 & 1.7 & 3.3 & 3.0 & 1.6 \\
$400 \mathrm{mg}(20-63 \mu \mathrm{m})$ & 700 & 4.1 & 3.3 & 3.0 & 1.6 \\
$600 \mathrm{mg}(20-63 \mu \mathrm{m})$ & 1190 & $5.9^{*}$ & 3.3 & 3.0 & 1.6 \\
$800 \mathrm{mg}(20-63 \mu \mathrm{m})$ & 1640 & $16.7^{*}$ & 3.3 & 3.0 & \\
\hline
\end{tabular}

Values with an asterisk indicate a high uncertainty

slow to sufficiently resolve the mass increase at the beginning. With a weight of $22 \mathrm{mg}$, both samples are on the lower limit of the measuring device. Hence, the reproducibility error and the error due to fluctuations in $\mathrm{RH}$ are comparatively large. Comparing the apparent fast mass increase for the $\mu \mathrm{m}$-samples with the markedly slower increase in the mm-samples supports the significance of a transport process at the beginning of the sorption kinetics in the low range of $\mathrm{RH}$. The long-time behaviour shows within the measurement error an identical small mass increase for the microtomed and grained sample until equilibrium condition is achieved. Within the measuring accuracy, this increase seems to be independent of the used sample geometry. Consequently, also for the $\mu \mathrm{m}$-range it seems as if there is no essential difference in the WVS behaviour of cut and grained wood, assuming that suited sample material is used.

An analysis with the PEK model gives good fitting results for both samples. The time constant $\tau_{1}$ of the fast process is lower, whereas the time constant $\tau_{2}$ for the slow process is even larger than in the previous results (Table 3). These high values for the characteristic times of the slow process could result from a mismatch of the model caused by the relatively large measurement error and a too slow gathering of measuring values for the mass increase of the samples. A failure of fitting the PEK model to grained wood with $20 \mathrm{mg}$ sample mass in the low RH range has also been reported in literature (Himmel and Mai 2016).

\section{Comparison of various grain sizes (monolayer)}

To point out the influence of grain size on the WVS behaviour of wood, the largest and smallest available grain sizes were used. Figure 6 shows the sorption kinetics for 
the $20-63 \mu \mathrm{m}$ and 500-1000 $\mu \mathrm{m}$ grain size distributions for a step change in RH of $0 \rightarrow 10 \%$ and $T=25^{\circ} \mathrm{C}$. The measurement error in the normalised representation is relatively large, as sample mass for approximately one monolayer of the smallest grain size is around $20 \mathrm{mg}$. A marked difference can be seen for the first measuring point (up to $10 \mathrm{~min}$ ), where the smaller grains show a faster mass increase than the larger ones. The long-time behaviour of the two samples shows within the measuring accuracy a similar small mass increase. According to the difference in grain size, these results point towards a transport limited process at the beginning of the mass increase. Either the pathways through the macrostructure, the higher accessibility of the cell wall material or the total amount of sorption sites per unit cross section could serve as a possible candidate for limiting the short-time kinetics. It should be mentioned that even if there is only cell wall material without any enclosed lumen pathways for the smallest grain size (i.e. the kinetic is for example limited by diffusion and adsorption of water molecules in the cell wall), there must be a reason why the larger grain size takes more time at the beginning of the mass increase than the smaller one. The difference in the WVS kinetics is much less than expected for a diffusion process with a factor of 20 difference in sample thickness (see, e.g., the results for the bound water diffusion model in Fig. 6b). This indicates processes like the accessibility of binding sites or the total amount of sorption sites per unit cross section to be more relevant in this stage. The results are compatible with the early experiments of Christensen and Kelsey (1959), where a small difference between the sorption kinetics of the $20 \mu \mathrm{m}$ microtomed slices and the $1 \mathrm{~mm}$ cut slice could

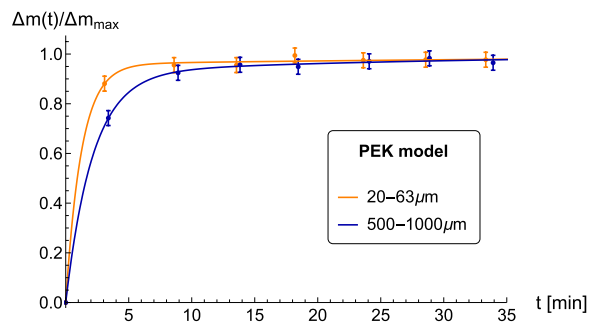

(a)

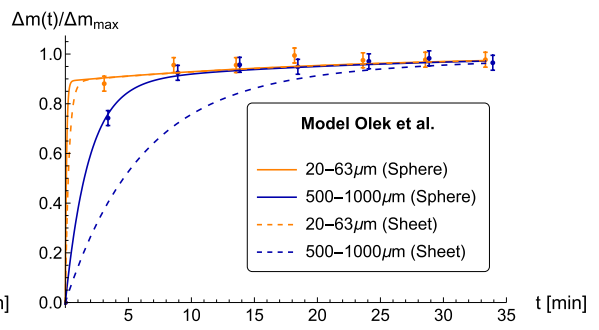

(b)

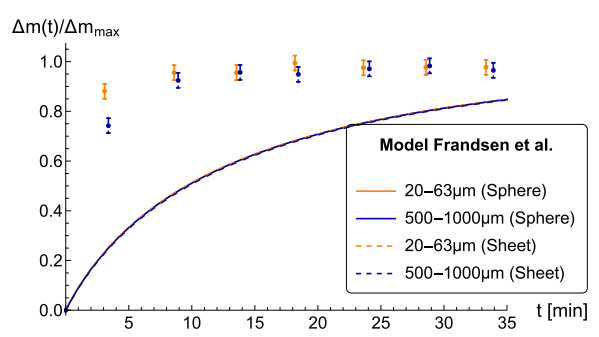

(c)

Fig. 6 Comparison of the WVS curves for monolayer grained wood with the largest (blue) and smallest grain size (orange). Markers indicate the measured values and solid/dashed lines the associated results of the simulations for the PEK model (a), the bound water diffusion model (b) and the coupled diffusion model (c) (color figure online) 
be seen for the lowest step change in RH, with the difference being much smaller than expected for the given sample lengths.

Regarding the modelling approaches, this experimental set-up serves as a possibility to verify the mentioned models in "Existing models" section for small sample sizes. The bound water diffusion model and the coupled diffusion model were thus solved for both, a homogeneous spherical and a sheet diffusion process. As could be seen in Fig. 6a, the PEK model could fit the two data sets very well. Analysing the parameters of the fitting result, the characteristic time for the fast process $\left(\tau_{1}\right)$ is similar to the $22 \mathrm{mg}$ samples (see Table 3 ). For the slow process the error of the fit results was relatively large and might explain the higher value of $\tau_{2}$ for the smaller grain size. The results for the bound water diffusion model are shown in Fig. 6b. The solid lines indicate the spherical solution and the dashed lines the sheet solution with the same parameters. As expected, the spherical solutions show a faster mass increase than the corresponding sheet solutions. A split-up between the two types of solutions can be seen for both grain sizes, even though it seems to be too large compared to the measured values. The time constant $\tau$ is comparable to the value for the slow process found with the PEK model and the surface emission factor $\sigma$ was kept constant for the two grain sizes (see Table 3). A variation for the latter would lead to an even larger split-up since smaller grains do have a larger surface. In contrast, the coupled diffusion model with the sorption term of Frandsen et al. (2007) shows a much slower mass increase of the simulation compared to the measurement (Fig. 6c). There is neither a difference between the sorption kinetics of the two grain sizes nor a visible distinction between the spherical and the sheet solution. This behaviour origins from the sorption term, which was initially constructed to yield similar results for samples with a thickness below roughly $1 \mathrm{~mm}$.

\section{Comparison of multilayer experiments}

\section{Comparison of grain size (multilayer)}

In order to get a better $S / N$-ratio of the monolayer experiment and to test the influence of grain size for multilayer experiments, three different grain sizes with an equal sample mass were compared. The results are shown in Fig. 7 for a sample mass of $200 \mathrm{mg}$ and a step change in $\mathrm{RH}$ of $0 \rightarrow 5 \%$ at $T=25^{\circ} \mathrm{C}$. A small difference can be seen at the beginning of the mass increase (up to $20 \mathrm{~min}$ ) with the larger grains showing a slightly slower increase than the smaller ones. For the long-time behaviour, a good congruence between the three grain size distributions is given until equilibrium condition is achieved. Similar results were also obtained with larger sample masses. Comparing with the monolayer results, it seems as if the influence of grain size (or cell wall accessibility) becomes less important for the multilayer experiments. Still, the grain layer thickness or the total amount of sorption sites per unit cross section seems to be of importance as the mass increase at the beginning of the multilayer experiments is markedly slower than in the monolayer case. The small differences in sheet thickness for the three grain sizes ("Experimental set-up" section) might thus be used to roughly estimate the differences in the 


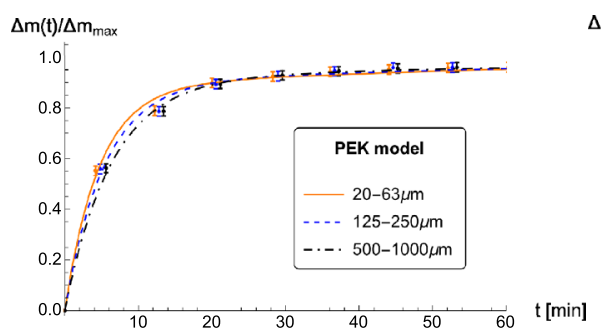

(a)

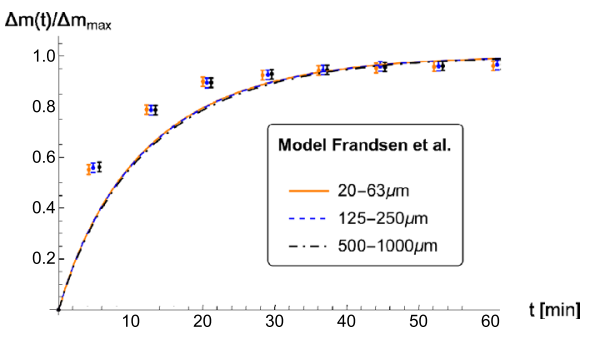

(c)

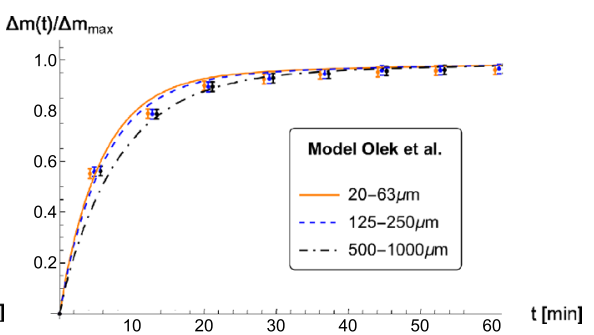

(b)

Fig. 7 Comparison of the WVS curves for multilayer grained wood with three different grain sizes and equal mass. Markers indicate the measured values and solid/dashed/dot-dashed lines the associated results of the simulations for the PEK model (a), the bound water diffusion model (b) and the coupled diffusion model (c)

WVS kinetics of grained wood with multiple grain layers. According to the similarity of the kinetic curves, it seems as if the given graining process has no significant impact on the WVS behaviour of wood. This includes any irregular graining losses among the various grain size distributions as also mechanical damage of the cell wall structure.

An adjustment of the PEK model on the multilayer experiments with equal mass is shown in Fig. 7a. The congruence between measured data and the fit results is not as good as in the case of the monolayer experiments. This might be due to a successive adsorption process of the grains or because transport of water vapour cannot be simplified (or neglected) above a certain sample thickness. Time constants are slightly increasing with grain size and are in the same order as in the mm-range (see Table 3). In comparison, the results of the bound water diffusion model for a 1D sheet diffusion process are shown Fig. 7b. A deviation can be seen at the beginning of the mass increase, whereas the long-time behaviour shows a good congruence to the measured values. According to the calculated grain layer thickness, the split-up between the smaller two grain sizes is appreciably lesser than for the largest grain size. Analogous to the monolayer experiments, surface emission factor was chosen to be identical for the three grain sizes and time constant $\tau$ is similar to the characteristic times for the slow process of the PEK model. Analysing the three multilayer samples with the coupled diffusion model yields a generally too slow mass increase for the simulations (Fig. 7c). The deviation is less than for the 
monolayer experiments, but still markedly larger compared to the other two modelling approaches. As previously mentioned this deviation arises due to a mismatch of the sorption term which dominates the initial mass increase particularly for thin samples. It seems as if the influence of sample thickness starts to become visible above $0.7 \mathrm{~mm}$ in this model.

\section{Comparison of grain layer thickness}

As the grain layer thickness (i.e. sample thickness) of grained wood seems to be important for the WVS kinetics, various masses with one grain size distribution were compared. Figure 8 shows the sorption kinetics for the smallest grain size $(20-63 \mu \mathrm{m})$ with a variation of sample thickness for a RH-step change of $0 \rightarrow 5 \%$ at $T=25^{\circ} \mathrm{C}$. An obvious difference between the four masses can be seen at the beginning, where thicker samples show a slower mass increase than thinner ones. Using a representation of the measured data over square root of time shows an approximately linear part for the mass increase at the beginning (Fig. 8d). This linear behaviour is characteristic for a diffusion process and is pointing towards diffusion being relevant for the short-time kinetics. However, the split-up seems to decrease with increasing grain layer thickness, indicating a deviation from a simple diffusion process. Similar results were also reported for cut wood (e.g. Wadsö 1993), where samples with various thickness do show a smaller split-up than expected for a diffusive process using Fick's law (Krabbenhoft and Damkilde 2004). In contrast, the long-time behaviour

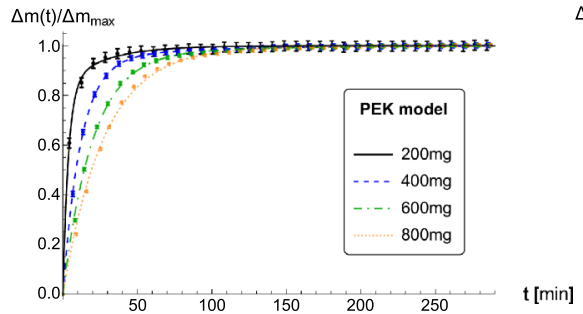

(a)

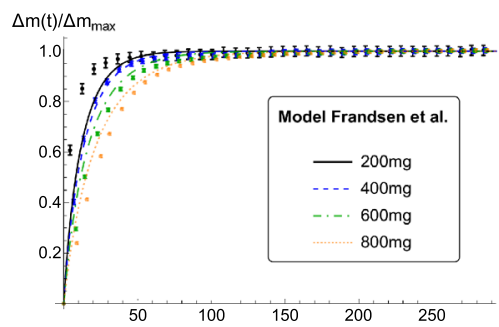

(c)

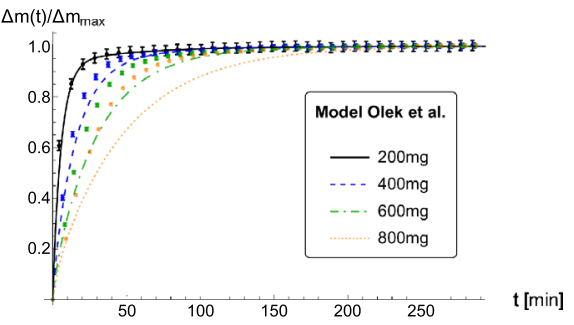

(b)

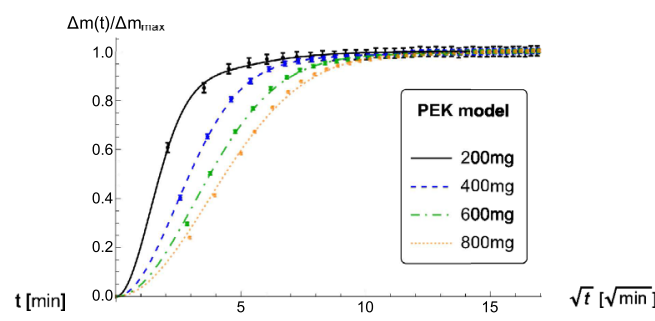

(d)

Fig. 8 Comparison of the WVS curves of grained wood with various grain layer thickness and one grain size $(20-63 \mu \mathrm{m})$. Markers indicate the measured values and solid/dashed/dot-dashed/dotted lines the associated results of the simulations for the PEK model (a), the bound water diffusion model (b) and the coupled diffusion model (c). Figure (d) shows additionally the measured values and the PEK simulations against $\sqrt{t}$ 
shows only small differences between the four samples, with a steeper slope for an increasing sample thickness. This seems likely for the consecutive humidification of single grains (or layers), as expected for a diffusive process through the multilayers. Hence, the consecutive adsorption of water $/ \mathrm{H}_{2} \mathrm{O}$-molecules of the grained samples initiates a successive starting of relaxation or reorganisation processes. The summation of all these processes thus leads to a delayed long-time mass increase for multilayer samples with a larger grain layer thickness. In a similar manner, the same mechanism should also hold for sliced wood with variable thickness. That is, the relaxation or reorganisation processes in the cell wall of wood are a local phenomenon initiated as soon as water vapour $/ \mathrm{H}_{2} \mathrm{O}$-molecules reach the given position. Such concepts of a local treatment of relaxation have already been mentioned in the literature (e.g. Engelund et al. 2012 and references therein) and requires a local sink as in the case of the coupled diffusion model. A global treatment of all these successive processes (as in the PEK model) seems thus to be an approximation, which might be reasonable merely for samples with a small thickness in longitudinal direction. For larger sample geometries, a local treatment of the relaxation and reorganisation processes with a corresponding equation for the water vapour supply appears to be necessary.

Concerning the modelling approaches, the four multilayer samples with different grain layer thicknesses could be captured with the PEK model as seen in Fig. 8a. The conformance is within the error bars for the $200 \mathrm{mg}$ sample but decreases with increasing sheet thickness (i.e. sample mass). Analysing the fit parameters of the model, both time constants show an increasing trend with increasing sample mass (Table 3). For the thickest sample, the time constant of the slow process seems to be too high, which might be caused by a mismatch of the model. Thus, the use of the PEK model seems to be limited for a small grain layer thickness of grained wood. With the previous results, it might also be concluded to use this model only for thin slices of cut wood, as for thicker samples the diffusion of water vapour through the macrostructure of wood has to be taken into account. An independent treatment of transport and relaxation seems therefore to be insufficient as both processes should depend on each other. Hence, a coupled description should provide the underlying framework for the PEK model. This would explain the importance of sample mass which has already been mentioned earlier (e.g. Xie et al. 2011). A rejection of the first few data points in the PEK fitting process (see e.g. Hill et al. 2010a; Sharratt et al. 2011; Popescu et al. 2015; Himmel and Mai 2016) might thus also be avoided. Figure $8 \mathrm{~b}$ shows the results of the bound water diffusion model. A split-up between the various layer thickness could be clearly seen, though it is too large compared to the measured values. Further, the decreasing differences for multilayer samples with a larger grain layer thickness cannot be captured with this model. Parameters were approximated to the $200 \mathrm{mg}$ sample and are identical to the previous experiment (see Table 3). In contrast, the results of the coupled diffusion model are given in Fig. 8c. A split-up among the multilayer samples with different grain layer thickness can now be observed, but with an increasing tendency for thicker samples. This increasing split-up is a consequence of the sorption term, which becomes less important above a certain sample thickness. The thinnest sample could not be captured as the sorption term serves as a lower limit for the mass increase in this model. 
Some criticism on the sorption term has also been mentioned in the literature (Eitelberger and Svensson 2012) and thus a modification of it might be fruitful.

\section{Conclusion}

The comparison of cut and grained wood showed a similar WVS behaviour in the $\mathrm{mm}$ and $\mu \mathrm{m}$-range, proving there are no principal differences in their sorption kinetics. For the cut and grained samples, it could be seen that thicker samples were appreciably slower in their short-time kinetics (i.e. mass increase at the beginning) than thinner ones, indicating a transport limited initial phase. In the same manner, monolayer experiments of grained wood exhibit a faster mass increase for smaller grains. Thus, transport effects are significant even for very small sample sizes. For multilayer experiments with equal sample mass, the influence of grain size seems to be less pronounced. A variation of grain layer thickness with equal grain size caused, however, a marked split-up in the short-time kinetics. Additionally, thinner samples showed a slightly faster equilibration in the long-time range than the thicker ones, which seems obvious for a successive adsorption of water vapour $/ \mathrm{H}_{2} \mathrm{O}$-molecules followed by a relaxation and reorganisation process. This trend holds even down to the smallest tested sample thickness $(20-63 \mu \mathrm{m})$ and is pointing towards a connection between the two stages. The WVS kinetics at low RH seems thus to be limited by a diffusion-like process at the beginning of the sorption kinetics and by a relaxation and reorganisation limited process for the long-time behaviour. Consequently, the second stage might be used to get insights into structural changes and rearrangements of water molecules, whereas the first stage seems to provide information about the penetration and support of water vapour $/ \mathrm{H}_{2} \mathrm{O}$-molecules to the sorption sites. Regarding the modelling approaches and their application to grained wood, a few general statements could be drawn:

PEK model: This study confirms the fast process of the PEK model being related to a transport limited process in the lower range of $\mathrm{RH}$. The measurements indicate diffusion of water vapour $/ \mathrm{H}_{2} \mathrm{O}$-molecules to the sorption sites being a possible candidate. Considering the long-time behaviour, the slow process in the PEK model was supported to be related to a relaxation and reorganisation limited process. Consequently, the independent treatment of both processes seems to be an approximation for small samples. A proper treatment of the transport processes and/or a coupled description appears to be advantageous and might avoid the exclusion of the first measurement points until target $\mathrm{RH}$ is reached.

Bound water diffusion model: The bound water diffusion model showed a too large split-up for the grain sizes (monolayer) and for the various grain layer thicknesses (multilayer). This seems to result as a consequence of both, treating the relaxation processes as a global phenomenon at the boundary and neglecting the water vapour transport inside and to the sample. Hence, a local treatment might provide better results when comparing similar samples with a variable thickness. For the long-time behaviour, this model leads to similar results as the PEK model, as both approaches use the same expression to treat relaxation and reorganisation processes. 
Coupled diffusion model: This model serves as the most comprehensive approach of the tested models as it accounts for the transport through the macrostructure, inside the cell wall and includes also the sorption process. Still, with the given sorption term and its parameters it led to the largest deviations to the experiments and the split-up for a variation of grain layer thickness could not be captured properly. A modification of this term might thus be worthwhile to treat the mass increase for samples with a thickness below $1 \mathrm{~mm}$.

Acknowledgements Open access funding provided by University of Innsbruck and Medical University of Innsbruck. This work has been founded by the Research Program Translational Research of the Standortagentur Tirol under the Project DigiPore $3 D$ and the Grant Doktoratsstipendium NEU aus der Nachwuchsförderung by the Universität Innsbruck, Vizerektorat für Forschung under the code 2015/2/ $T E C H-26$. Their financial support is gratefully acknowledged.

Open Access This article is distributed under the terms of the Creative Commons Attribution 4.0 International License (http://creativecommons.org/licenses/by/4.0/), which permits unrestricted use, distribution, and reproduction in any medium, provided you give appropriate credit to the original author(s) and the source, provide a link to the Creative Commons license, and indicate if changes were made.

\section{References}

Alpay E, Scott D (1992) The linear driving force model for fast-cycle adsorption and desorption in a spherical particle. Chem Eng Sci 47(2):502-504

Avramidis S, Siau JF (1987) An investigation of the external and internal resistance to moisture diffusion in wood. Wood Sci Technol 21(3):249-256

Christensen GN (1965) The rate of sorption of water vapor by thin materials. In: Wexier A (ed) Humidity and moisture, vol 4. Reinhold Publ, New York, pp 279-293

Christensen G (1967) Sorption and swelling within wood cell walls. Nature Publishing Group, London, pp 782-784

Christensen G, Kelsey K (1959) Die Geschwindigkeit der Wasserdampfsorption durch Holz [The rate of sorption of water vapour by wood]. Holz Roh- Werkst 17(5):178-188

Crank J (1975) The mathematics of diffusion, 2nd edn. Oxford University Press, Oxford

Eitelberger J, Svensson S (2012) The sorption behavior of wood studied by means of an improved cup method. Transp Porous Media 92(2):321-335

Eitelberger J, Hofstetter K, Dvinskikh SV (2011) A multi-scale approach for simulation of transient moisture transport processes in wood below the fiber saturation point. Compos Sci Technol 71(15): 1727-1738

Engelund ET, Thygesen LG, Svensson S, Hill CAS (2012) A critical discussion of the physics of woodwater interactions. Wood Sci Technol 47(1):141-161

Foley N, Thomas K, Forshaw P (1997) Kinetics of water vapor adsorption on activated carbon. Langmuir 13(7):2083-2089

Frandsen HL, Damkilde L, Svensson S (2007) A revised multi-Fickian moisture transport model to describe non-Fickian effects in wood. Holzforschung 61(5):563-572

Glass SV, Boardman CR, Zelinka SL (2017) Short hold times in dynamic vapor sorption measurements mischaracterize the equilibrium moisture content of wood. Wood Sci Technol 51(2):243-260

Hartley ID, Kamke A, Va B (1992) Cluster theory for water sorption in wood. Wood Sci Technol 99:83-99

Hill CAS, Norton A, Newman G (2010a) Analysis of the water vapour sorption behaviour of Sitka spruce based on the parallel exponential kinetics model. Holzforschung 64:469-473

Hill CAS, Norton AJ, Newman G (2010b) The water vapour sorption properties of Sitka spruce determined using a dynamic vapour sorption apparatus. Wood Sci Technol 44(3):497-514

Hill CAS, Moore J, Jalaludin Z, Leveneu M, Mahrdt E (2011) Influence of earlywood/latewood and ring position upon water vapour sorption properties of Sitka spruce. Int Wood Prod J 2(1):12-19 
Himmel S, Mai C (2016) Water vapor sorption of wood modified by acetylation and formalizationanalyzed by a sorption kinetics model and according to modeless thermodynamic considerations. Holzforschung 70(3):203-213

Kohler R, Dück R, Ausperger B, Alex R (2003) A numeric model for the kinetics of water vapor sorption on cellulosic reinforcement fibers. Compos Interfaces 10(2-3):255-276

Krabbenhoft K, Damkilde L (2004) A model for non-Fickian moisture transfer in wood. Mater Struct 37(November):615-622

Okubayashi S, Griesser UJ, Bechtold T (2004) A kinetic study of moisture sorption and desorption on lyocell fibers. Carbohydr Polym 58(3):293-299

Olek W, Weres J (2007) Effects of the method of identification of the diffusion coefficient on accuracy of modeling bound water transfer in wood. Transp Porous Media 66(1-2):135-144

Olek W, Perré P, Weres J (2005) Inverse analysis of the transient bound water diffusion in wood. Holzforschung 59(1):38-45

Olek W, Perré P, Weres J (2011) Implementation of a relaxation equilibrium term in the convective boundary condition for a better representation of the transient bound water diffusion in wood. Wood Sci Technol 45(4):677-691

Popescu CM, Hill CAS (2013) The water vapour adsorption-desorption behaviour of naturally aged Tilia cordata Mill. wood. Polym Degrad Stab 98(9):1804-1813

Popescu CM, Hill CAS, Curling S, Ormondroyd G, Xie Y (2014) The water vapour sorption behaviour of acetylated birch wood: how acetylation affects the sorption isotherm and accessible hydroxyl content. J Mater Sci 49(5):2362-2371

Popescu CM, Hill CAS, Anthony R, Ormondroyd G, Curling S (2015) Equilibrium and dynamic vapour water sorption properties of biochar derived from apple wood. Polym Degrad Stab 111:263-268

Sharratt V, Hill CAS, Zaihan J, Kint DP (2011) The influence of photodegradation and weathering on the water vapour sorption kinetic behaviour of scots pine earlywood and latewood. Polym Degrad Stab 96(7):1210-1218

Siau JF (1984) Transport processes in wood. Springer, Berlin

Wadsö L (1993) Measurements of water vapour sorption in wood. Wood Sci Technol 28(1):59-65

Xie Y, Hill CAS, Jalaludin Z, Sun D (2011) The water vapour sorption behaviour of three celluloses: analysis using parallel exponential kinetics and interpretation using the Kelvin-Voigt viscoelastic model. Cellulose 18(3):517-530

Zaihan J, Hill CAS, Curling S, Hashim WS, Hamdan H (2009) Moisture adsorption isotherms of Acacia mangium and Endospermum malaccense using dynamic vapour sorption. J Trop For Sci 21(3):277-285 